\title{
Anomalies of the Aortic Arch in Dogs: Evaluation with the use of Multidetector Computed Tomography and Proposal of an advanced Classification Scheme
}

\section{Christiane Schorn ( $\nabla$ christiane.schorn@web.de)}

Department of Veterinary Clinical Sciences, Small Animal Clinic, University of Giessen, Germany Nicolai Hildebrandt

Department of Veterinary Clinical Sciences, Small Animal Clinic, University of Giessen, Germany Sebastian Schaub

Department of Veterinary Clinical Sciences, Small Animal Clinic, University of Giessen, Germany

\section{Research Article}

Keywords: computed tomography (CT), vascular ring

Posted Date: May 6th, 2021

DOl: https://doi.org/10.21203/rs.3.rs-477812/v1

License: (c) (1) This work is licensed under a Creative Commons Attribution 4.0 International License. Read Full License 
Anomalies of the Aortic Arch in Dogs: Evaluation with the use of Multidetector Computed

Tomography and Proposal of an advanced Classification Scheme

\section{Authors}

Christiane Schorn, Dr. med. vet.

Justus-Liebig University Clinic for Small Animals, Frankfurter Straße 114, 35390 Gießen

Nicolai Hildebrandt, Dr. med. vet.

Justus-Liebig University Clinic for Small Animals, Frankfurter Straße 114, 35390 Gießen

Sebastian Schaub, Dr. med. vet.

Justus-Liebig University Clinic for Small Animals, Frankfurter Straße 114, 35390 Gießen

\section{Corresponding Autor}

Christiane Schorn, Dr. med. vet.

\section{ABSTRACT}

Background: Congenital anomalies of the aortic arch are important to recognize as they may be associated with vascular rings. The most common vascular ring anomaly in dogs is persistent right aortic arch. However, published data of the distribution and percentage of the different types of vascular rings and other aortic arch anomalies are lacking. The objective of this retrospective descriptive study was to characterize the amount and types of aortic arch anomalies that can be detected in thoracic computed tomography (CT) examination in dogs. Results: Dogs that underwent thoracic CT between 2008 and 2020 were included. A total of 213 CT studies were sampled; 21 dogs $(21 / 213,9.9 \%)$ showed a right aortic arch, and the 
following additional findings were detected: aberrant left subclavian artery $(17 / 21,76.2 \%)$ branching from the PDA (1/21, 4.8\%), left-sided brachiocephalic trunk (3/21, $14.3 \%)$, bicarotid trunk (17/21, 81.0\%), double aortic arch (1/21, 4.8\%). No dog presented right aortic arch without further anomalies. Also, 192 dogs (192/213, 90.1\%) showed left aortic arch. The following additional abnormalities were obtained: aberrant right subclavian artery (3/192, 1.6\%) without clinical signs of vascular ring, aberrant vessel branching from the aorta into the left caudal lung lobe $(2 / 192,1.0 \%)$, focal dilataion of the left or right subclavian artery $(2 / 192$, 1.0\%), bicarotid trunk (1/192, 0.5\%).

Conclusion: The current study indicates that aberrant left subclavian artery is the most common additional finding in dogs with persistent right aortic arch and that left-sided brachiocephalic trunk can occur in dogs, which was previously unpublished. Aberrant right subclavian artery can be an incidental CT finding without clinical relevance.

\section{BACKGROUND}

Anomalies of the aortic arch are important to recognize because they may be associated with vascular rings (1). A vascular ring is defined as a congenital disorder of the aortic vasculature that involves complete or partial encircling of the esophagus and trachea with secondary esophageal compression (1-3). Due to esophageal compression, affected dogs show postprandial regurgitation of solid food (3). Contrast-enhanced computed tomography allows an accurate anatomic diagnosis of vascular anomalies of the aortic arch $(4,5,6)$. Persistent right aortic arch is one of the most common ring anomalies in dogs, with a prevalence of about $7 \%$ $(7,8)$. Pure-breed dogs seem to be more often affected than mixed-breed dogs, with German Shepherd dogs being overrepresented $(7,8)$. Embryologically, the aorta develops out of an aortic sac, which is connected to the bilateral dorsal aortae by six paired aortic arches that 
develop bilaterally to the pharynx (9). The dorsal aortae form a ring. During embryogenesis, the first, second, and fifth aortic arch degenerate. The third arches bilaterally become the common carotid arteries, and the dorsal aortae between the third and fourth arch degenerate $(9,10)$. The left fourth aortic arch forms the ascending aorta, and the right fourth aortic arch contributes, together with the seventh intersegmental artery, to the right subclavian artery $(9,10)$. The brachiocephalic trunk originates from the aortic sac and the third and fourth aortic arch. The sixth aortic arches become the left and right pulmonary arteries and continue as left and right ductus arteriosus. The right ductus arteriosus disappears prenatally, the left ductus arteriosus closes postnatally, and the ligamentum arteriosum remains (10). The right dorsal aorta degenerates caudally to the right subclavian artery, and the left dorsal aorta forms the descending aorta (9). When the right fourth aortic arch and the right dorsal aorta enlarge instead of the left, persistent right aortic arch develops (10). Physiologically, the right ductus arteriosus degenerates, and the left ductus arteriosus persists, forming a connection between the left pulmonary artery and the abnormal right aortic arch, leading to constriction of the esophagus (10). This is the most common type of vascular ring anomaly in dogs, classified as type one (9). Other described ring anomalies in dogs include a double aortic arch, persistent right ductus arteriosus, aberrant left subclavian artery (usually seen in combination with right aortic arch), and aberrant right subclavian artery (10). Because of the increased availability of computed tomography (CT), more thoracic CT studies are available, and aortic arch anomalies can be detected even in cases without clinical signs of vascular ring anomaly. To the authors knowledge, there is no data describing the frequency of aortic arch anomalies that could be detected during standard computed tomography of the thoracic cavity. Therefore, the purpose of the study was to retrospectively 
evaluate thoracic CT studies and to evaluate the percentage and types of aortic arch anomalies found in dogs.

\section{MATERIAL AND METHODS}

The study used a retrospective, case series descriptive design. As a retrospective study, all data sets were acquired from clinical patients using standard veterinary practice, and no animal care and use protocol was required. Thoracic CT examinations of dogs between 2008 and 2020 were searched using the database of the Justus-Liebig University Clinic for small animals. Only dogs with a complete dataset, including a post-contrast study, were included. For dogs meeting the inclusion criteria, the following medical record data were documented: breed, sex, age at time of imaging, date of imaging, and reason for presentation.

\subsection{CT examination}

The computed tomographic examinations were performed under general anesthesia, which was induced using conventional techniques and maintained with isoflurane by use of mechanical ventilation. All CT examinations were carried out using a 16-detector-row computer tomography system (either SOMATOM Emotion, Siemens Healthcare, Erlangen Germany, or Diamond Select Brilliance, Philips Health Systems, Best, Netherlands). The CT scans were performed in sternal recumbency with breath-hold technique whenever possible. Contrast medium (AccupaqueTM 300; GE Healthcare Buchler GmbH \& Co. KG; Braunschweig; Germany) was injected intravenously at a dose of $2 \mathrm{mg} / \mathrm{kg}$, using a power injector (Medtron AG, Saarbrücken, Germany) at a maximum flow rate of $5 \mathrm{ml} / \mathrm{sec}$, followed by a saline flush at the same injection rate. In most cases, scans were delayed for 60 to 90 seconds after contrast medium administration. In some cases, arterial phase images were acquired using bolus 
tracking. Scanner setting was as follows: $1.5 \mathrm{~mm}$ slice thickness, a pitch of 0.8 , tube rotation time $0.6 \mathrm{~s}, 130 \mathrm{kV}$, and 160 to $200 \mathrm{~mA}$.

\subsection{Image evaluation}

All data sets were reviewed by a first-year resident (C.S.) of the European College of Veterinary Diagnostic Imaging (ECVDI) and one ECVDI board-certified radiologist (S.S.), using the DICOMviewing software (Horos v. 3.3.6, Los Angeles, California). Image orientation, window width and level could be adjusted by the reviewers according to personal preference.

Abnormalities evaluated were limited to the aortic arch. Each case was evaluated for pathologies concerning the aortic arch, including the right aortic arch, the existence of a right or left aberrant subclavian artery, patent ductus arteriosus (PDA), normal anatomy of the brachiocephalic trunk, and other findings.

\section{RESULTS}

A total of 213 thoracic CT scans where evaluated. A group of twenty-one dogs presented due to clinical signs referring to a ring anomaly, at least regurgitation after food intake. Two dogs presented due to exercise intolerance. The remaining 192 dogs underwent thoracic CT due to other reasons not related to vascular rings, such as spontaneous pneumothorax, lung diseases, or for metastatic screening.

\subsection{Dogs with persistent right aortic arch}

All 21 dogs presenting with clinical signs of suspected vascular ring anomaly showed a right aortic arch. There were 15 female and 6 male dogs of the following breeds: 9 Labrador Retrievers, 4 German Shepherds, 2 mixed-breed dogs, 1 French Bulldog, 1 Gos d'Atura Català, 
1 Husky, 1 Jack Russel Terrier, 1 Border Collie, and 1 Australian Shepherd. The mean age of the dogs was 2 months (range 6 weeks to 4 months).

None of the dogs with a right aortic arch showed a physiological brachiocephalic trunk.

The following abnormal aortic branching patterns were found (Table 1):

1.) 17 dogs showed a bicarotid trunk branching from the right aortic arch: in the remaining 2 dogs, the left and right carotid artery arose separately from the right aortic arch;

2.) 16 dogs showed an aberrant left subclavian artery, crossing the esophagus dorsally from the right arch to the left side;

3.) one dog with a patent ductus arteriosus showed the left subclavian artery branching from the ductus arteriosus and mild dilation of the left subclavian artery;

4.) three dogs had a left-sided brachiocephalic trunk composed of both carotid arteries and the left subclavian artery, and the right subclavian artery arose separately from the aorta;

5.) one dog had a double aortic arch; in this case, the left subclavian artery branched from the left aortic arch, the right subclavian artery, and a bicarotid trunk branched from the right aortic arch;

5.) in all cases, the right subclavian artery had a discrete branch from the right aortic arch.

TABLE 1 Percentages of additional findings in dogs with persistent right aortic arch.

\begin{tabular}{|l|l|l|}
\hline Anomalies in dogs with persistent right aortic arch $(\mathbf{n}=\mathbf{2 1})$ & Dogs & Percentage \\
\hline Physiological brachiocephalic trunk & 0 & 0.0 \\
\hline Aberrant left subclavian artery & 16 & 76.2 \\
\hline Bicarotid trunk & 17 & 81.0 \\
\hline $\begin{array}{l}\text { Left-sided brachiocephalic trunk including both carotid arteries } \\
\text { and the left subclavian artery }\end{array}$ & 3 & 14.3 \\
\hline \begin{tabular}{l} 
Aberrant subclavian artery branching from the PDA \\
\hline
\end{tabular} & 1 & 4.8 \\
\hline
\end{tabular}




\subsection{Aortic arch anomalies in dogs with left aortic arch}

Two of the remaining 192 dogs presented due to exercise intolerance. Dog signalment was one male 2-month-old Flat-Coated Retriever and one male 6-month-old Shetland Sheepdog. The CT examination revealed a congenital malformation of the aortic arch, with aberrant origin of an arterial vessel, arising from the aorta at the level of the fourth thoracic vertebral body. The aberrant vessel was running caudally and ventrally, draining into the left caudal lung lobe with subsequent systemic to pulmonary connection. Both dogs showed a persistent ductus arteriosus. The Flat-Coated Retriever presented a hypoplastic pulmonary artery on the left side as well as a moderately reduced lung volume on the left side.

The remaining 190 dogs showed no signs of cardiovascular disease. The mean age of the dogs was 6.4 years (range 1 to 16 years). There were 47 female, 42 female-spayed, and 56 male and 45 male-neutered dogs, representing 61 different breeds: 153 were pure-breed dogs and 37 were mixed-breed ones. The following CT findings regarding the aortic arch were documented (Table 2):

1.) All dogs had a left aortic arch;

2.) three dogs showed an aberrant right subclavian artery arising as a separate vessel from the descending aorta and passing the esophagus dorsally;

3.) one dog showed focal moderate dilation of the left subclavian artery;

4.) one dog showed focal moderate dilation of the right subclavian artery;

5.) one dog showed a bicarotid trunk arising from the brachiocephalic trunk. 
TABLE 2 Percentages of aortic arch anomalies found in dogs without persistent right aortic arch.

\begin{tabular}{|l|l|l|}
\hline $\begin{array}{l}\text { Aortic arch anomalies in dogs without persistent right aortic } \\
\text { arch ( } \mathbf{n}=\mathbf{1 9 2} \text { ) }\end{array}$ & Dogs & Percentage \\
\hline $\begin{array}{l}\text { Malformation of the aortic arch on the left side with aberrant } \\
\text { vessel draining into the left caudal lung lobe }\end{array}$ & 2 & 1.0 \\
\hline Aberrant right subclavian artery & 3 & 1.6 \\
\hline Focal dilatation of the left subclavian artery & 1 & 0.5 \\
\hline Focal dilatation of the right subclavian artery & 1 & 0.5 \\
\hline Bicarotid trunk arising from the brachiocephalic trunk & 1 & 0.5 \\
\hline
\end{tabular}

\section{DISCUSSION}

To the authors' knowledge, this is the first paper systematically describing variations of the aortic arch that can be found during thoracic CT examination in dogs. Concerning the whole study population, $9.9 \%$ showed a persistent right aortic arch; these findings are consistent with previously published data $(7,8)$. In contrast to other studies, German Shepherd dogs were not overrepresented in the study population $(6,7)$. The most affected breed described in the current paper was the Labrador Retriever dog, representing $42.9 \%$ of the cases. In current literature, seven different types of vascular ring anomalies have been classified in dogs: Type 1, consisting of persistent right aortic arch with persistent left ligamentum arteriosum; Type 2, consisting of persistent right aortic arch with persistent left subclavian artery; Type 3, consisting of persistent right aortic arch with persistent left ligamentum arteriosum and left subclavian artery; Type 4, consisting of double aortic arch; Type 5, consisting of normal left aortic arch with persistent right ligamentum arteriosum; Type 6, consisting of normal left aortic arch with persistent right subclavian artery; Type 7, consisting of normal left aortic arch with persistent right ligamentum arteriosum and right subclavian artery (9). In a previously published review, two additional types of vascular ring anomalies have been mentioned (3). The first has been characterized in a single surgical case report in 1979; the authors described 
an aberrant branch of the aorta arising 3 centimeters caudal to the left subclavian artery, passing anteromedially and draining into the brachiocephalic artery (11). Considering the long course of the described vessel, a complete vascular ring appears less likely, and the complete and correct surgical visualization of the vessel might be questionable. The second additional mentioned type of vascular ring has also been found surgically and described as incomplete esophageal constriction due to the first right intercostal arteries branching directly dorsal from the aorta instead of branching from the costocervical trunk (12). Considering the incomplete vascular ring in both cases, the two proposed additional types represent rather aberrant aortic vessels than classical ring anomalies. Therefore, they were not included into the classification scheme proposed in the current article. Concerning the own study population, Type 3 was the most frequently detected ring anomaly, affecting $76.2 \%$ of all dogs with right aortic arch. Type 1 anomaly, without additional vascular changes, was not detected in any dog. his is in contrast to previously published data describing Type 1 as the most common type (9). Embryologically, right aortic arch results from regression of the left fourth arch between the left carotid artery and the left subclavian artery, resulting in the left subclavian artery being the last one branching from the aorta (1). Type 1 is therefore unlikely to occur. In a more recently published study, an aberrant left subclavian artery was detected in $33 \%$ of the cases $(4,13)$. The lower percentages of aberrant left subclavian artery, published in older literature, are probably because the final diagnosis was exclusively made by surgery. In some dogs, retroesophageal left subclavian artery as well as other additional vascular anomalies are not obvious and may not have been identified by the surgeon. Another study describing CT features of dogs with right aortic arch revealed an aberrant left subclavian artery in $60 \%$ of the cases; in the current study, additional vascular changes of the persistent right aortic arch were present in all dogs (4). Therefore, CT examination appears to be more 
accurate in detecting an aberrant left subclavian artery than surgery alone. Surgery may underestimate additional vascular abnormalities. The clinical relevance of an aberrant left subclavian artery is unclear and may not contribute to esophageal compression, probably because of its more dorsally and near-midline origin (6). Nevertheless, surgeons should be aware of the presence of aberrant left subclavian artery to assess the possible constriction during thoracotomy.

Type 6 anomaly was detected in three dogs without clinical signs suspicious for vascular ring anomaly and occurred as an incidental finding in the CT examination of the thoracic cavity performed due to other reasons. Aberrant right subclavian artery is usually associated with normal left aortic arch (10). Embryologically aberrant right subclavian artery occurs when the right dorsal aorta, cranial to the subclavian artery, abnormally degenerates. Consequently, the distal right aorta, instead of the right fourth arch, becomes the base of the right subclavian artery, which is linked caudally to the left aorta. Therefore, the right aberrant subclavian artery is the last branch leaving the aortic arch $(1,14)$. In human medicine, left aortic arch with aberrant right subclavian artery is the most common congenital malformation of the aortic arch, with a prevalence described between 0.5 and $2 \%$, leading to esophageal compression in about $10 \%$ of the cases $(1,15)$. In veterinary medicine, only few case reports exist, and there are no data describing the overall prevalence $(14,16-19)$. The clinical relevance of aberrant right subclavian artery in dogs is unclear, with some case reports describing clinical syndromes such as dysphagia and regurgitation (11,13-16). The data collected in the present study show that an aberrant right subclavian artery in dogs, as it is reported in humans, can be an incidental finding without any clinical relevance. The prevalence of an aberrant right subclavian artery is $1.4 \%$ in the study population. Another incidental finding was a mild focal dilatation of the right and left subclavian artery, detected in two dogs without any clinical 
relevance. In human medicine, dilatation of the subclavian artery is defined as Kommerell's diverticulum and characterized by focal dilatation near the origin from the aorta $(4,20,21)$. The dilatation of the subclavian artery recognized in the own population was more distally and can therefore not be defined as Kommerell's diverticulum, but may be assessed as a normal anatomical variant.

One main finding of the study was the left-sided brachiocephalic trunk, found in three dogs with right aortic arch. The left-sided brachiocephalic trunk was composed of the left subclavian artery and both carotid arteries and thus represented a complete reflection of the brachiocephalic trunk on the left side. To our knowledge, this type has not been previously described in dogs. In human medicine, this type has been described as right aortic arch with mirror image branching and is the second most common form of a right-sided aortic arch (1). Embryologically, the left-sided brachiocephalic trunk results from regression of the left dorsal aorta distal to the origin of the seventh intersegmental artery (1). The findings of the current study show that a left-sided brachiocephalic trunk also occurs in dogs, with a prevalence of $14.3 \%$ of dogs with right aortic arch in the study population. Therefore, complementing the current classification scheme by the new type is proposed (Fig. 1).

One dog with right aortic arch showed an aberrant left subclavian artery branching from the patent ductus arteriosus. This type of aortic malformation has been described in only one dog before and thus appears to represent a rare variant (22). The malformation, which is defined by an isolated left subclavian artery, is caused by regression of the left arch at two segments cranially and caudally to the left subclavian artery (1). Like the previously reported type, this variant has not yet been characterized and should be included in the new modulated classification scheme, which is proposed by the authors (Fig. 1). Regarding surgical treatment, in both cases, ligation and dissection of the left subclavian artery were performed. In human 
medicine, occlusion or stenosis of the proximal subclavian artery results in reversal blood flow through the vertebral artery. Common clinical signs are vertigo, syncope, and intermittent claudication of the involved upper extremity; the syndrome is known as subclavian steel syndrome (23). No clinical signs consistent with the described subclavian steel syndrome were detected in one of the two dogs following surgery. Nevertheless, surgeons should be aware of potential side effects, and dissection of the subclavian artery with subsequent anastomosis to the left carotid artery could be recommended to prevent subclavian steel syndrome.

Two dogs in our study showed an aberrant vessel branching from the aortic arch, coursing caudally and draining into the left caudal lung lobe. Systemic to pulmonary shunting vessels have been described in dogs in multiple case reports (24-29). In most cases, in the veterinarian literature, systemic to pulmonary shunting is described as hypertrophy of the bronchoesophageal artery, with multiple tortuous shunt vessels (24-27). Bronchoesophageal artery hyperplasia can be a congenital disorder or may be acquired due to long-standing hypoxic states or due to pulmonary artery flow reduction $(27,30)$. The two patients in the current study showed no evidence of bronchoesophageal artery hyperplasia, and there was only a single linear shunt vessel visible, branching directly from the descending aorta. In the respective patients, both pulmonary arteries were physiologically detectable even though the left pulmonary artery in one dog was mildly hypoplastic. In contrast to other authors, no direct connection between the shunt vessel and the pulmonary artery was detected (31). There was no evidence for acquired aortic to pulmonary shunt vessels in the current study population and, except for the PDA, no accompanying congenital cardiac anomalies were evident. In human medicine, persistence of the $5^{\text {th }}$ aortic arch can lead to systemic to pulmonary connection by an aberrant vessel $(1,32,33)$. Physiologically, the $5^{\text {th }}$ aortic arches are rudimentary vessels that quickly degenerate during embryogenesis and lie between the $4^{\text {th }}$ 
and $6^{\text {th }}$ aortic arch $(1,33)$. As the herein described anomalous vessels originated from the descending aorta caudal to the PDA, representing a remnant of the $6^{\text {th }}$ aortic arch, a persistent $5^{\text {th }}$ aortic arch is considered unlikely in these cases. Therefore, the origin of the described vessels remains unclear and most likely represents an aberrant intercostal artery. The aberrant vessel in the 6-month-old Shetland Sheepdog was closed via catheter embolization. The second dog was lost to follow-up.

Limitations of this study include the retrospective nature. Arterial phase-computed tomographic studies were not available in all cases because thoracic-computed tomography was often performed due to other reasons than suspected vascular abnormalities. Nevertheless, visualization of the aortic arch was good in the late-phase contrast study. The small group of dogs with right aortic arch did not allow generating epidemiological data in respect of the distribution of congenital aortic arch malformation in dogs. The own referring clinic has a large focus on cardiac patients, and therefore, patients with cardiac diseases may have been overrepresented in the current study population.

\section{CONCLUSIONS}

This study describes aortic arch anomalies and their distribution in a large population of dogs, detected during thoracic-computed tomography. The findings indicate that further classification of right aortic arch types is needed since two more - to date unclassified - types are present in dogs, leading to a total number of at least nine different types in dogs. A new classification scheme is therefore proposed by the authors. Furthermore, the results indicate that the aberrant right subclavian artery is an incidental finding in dogs with left aortic arch without evidence of clinical signs. Future studies are needed to determine whether the findings from this study remain valid even for a larger dog population. 


\section{Figure Legend}

\section{Figure 1}

Title: Classification scheme for aortic arch anomalies

Legend: (A) normal anatomy of the aortic arch; (B) Type 1, consisting of persistent right aortic arch with persistent left ligamentum arteriosum; (C) Type 2, consisting of persistent right aortic arch with persistent left subclavian artery; (D) Type 3, consisting of persistent right aortic arch with persistent left ligamentum arteriosum and left subclavian artery; (E) Type 4, consisting of double aortic arch; (F) Type 5, consisting of normal left aortic arch with persistent right ligamentum arteriosum; (G) Type 6, consisting of normal left aortic arch with persistent right subclavian artery; $(\mathrm{H})$ Type 7, consisting of normal left aortic arch with persistent right ligamentum arteriosum and right subclavian artery; (I) Type 8, consisting of right aortic arch with left subclavian branching from the left sided ligamentum arteriosum; (J) Type 9, consisting of right sided aortic arch with "mirror" image.

\section{Declarations}

Ethics approval and consent to participate: As a retrospective study, all data sets were acquired from clinical patients using standard veterinary practice, and no animal care and use protocol was required. All patient owners provided written informed consent prior to enrolment in the study.

Consent for publication: not applicable

Availability of data and materials: The datasets analyzed during the current study are available from the corresponding author upon reasonable request.

Competing interests: The authors declare that they have no competing interests.

Funding: not applicable 
Authors' contributions: C.S. and S.S. analyzed and interpreted the patient data sets and mainly contributed to the writing of the manuscript. N.H. contributed to the patient selection. All authors have read and critically reviewed the manuscript and provided feedback on drafts, as well as approved the final version of the manuscript.

Acknowledgements: not applicable

Authors' information: not applicable

\section{References}

1. Hanneman K, Newman B, Chan F. Congenital variants and anomalies of the aortic arch. Radiographics. 2016;37(1):32-51.

2. Gould SW, Rigsby CK, Donnelly LF, McCulloch M, Pizarro C, Epelman M. Useful signs for the assessment of vascular rings on cross-sectional imaging. Pediatr Radiol. 2015;45(13):2004-16.

3. Morgan KRS, Bray JP. Current diagnostic tests, surgical treatments, and prognostic indicators for vascular ring anomalies in dogs. J Am Vet Med Assoc [Internet]. 2019 Mar 15;254(6):728-33. Available from: https://doi.org/10.2460/javma.254.6.728

4. Henjes CR, Nolte I, Wefstaedt P. Multidetector-row computed tomography of thoracic aortic anomalies in dogs and cats: Patent ductus arteriosus and vascular rings. Bmc Vet Res. 2011;7(1):57.

5. Joly H, D'Anjou M, Huneault L. IMAGING DIAGNOSIS-CT Angiography od a rare vascular ring anomaly in a dog. Vet Radiol Ultrasoun. 2008;49(1):42-6.

6. Pownder S, Scrivani PV. Non-selective computed tomography angiography of a vascular ring anomaly in a dog. J Vet Cardiol. 2008;10(2):125-8.

7. Patterson DF. Canine congenital heart disease: epidemiology and etiological hypotheses. J Small Anim Pract. 1971;12(5):263-87.

8. Patterson DF. Epidemiologic and genetic studies of congenital heart disease in the dog. Circ Res. 1968;23(2):171-202.

9. Helphrey ML. Vascular ring anomalies in the dog. Vet Clin North Am Small Animal Pract. 1979;9(2):207-18.

10. Ellison G. Vascular ring anomalies in the dog and cat. Compend Contin Educ Vet -North American Edition-. 1980 Feb 1;2:693-705.

11. Holmberg DL, Presnell KR. Vascular ring anomalies: Case report and brief review. CAN VET J. 1979 Mar 1;20(3):78-81. Available from: https://pubmed.ncbi.nlm.nih.gov/436102 
12. Bellenger CR, Warren DF. Esophageal constriction due to aberrant vessels in dogs. Mod Vet Pract. 1970;(71):51-3.

13. Buchanan JW. Tracheal signs and associated vascular anomalies in dogs with persistent right aortic arch. J Vet Intern Med. 2004 Jul-Aug;18(4):510-4.

14. Kleer VS de. An anomalous origin of the right subclavian artery in the dog. Brit Vet J. 1971;127(2):76-82.

15. Türkvatan A, Büyükbayraktar FG, Ölçer T, Cumhur T. Congenital anomalies of the aortic arch: Evaluation with the use of multidetector computed tomography. Korean J Radiol. 2009;10(2):176-84.

16. Miller R, Wilson C, Wray J, Jakovljevic S, Tappin S. Adult-onset regurgitation in a dog with an aberrant right subclavian artery: a CT angiographic study. Vet Rec Case Reports. 2015;3(1):e000138.

17. Muldoon M, Birchard S, Ellison G. Long-term results of surgical correction of persistent right aortic in dogs: 25 Cases (1980-1995). JAVMA-J AM VET MED A. 1997 Jul 1;210:1761-3.

18. Yoon $\mathrm{H}-\mathrm{Y}$, Jeong $\mathrm{S}$. Surgical correction of an aberrant right subclavian artery in a dog. CAN VET J. 2011 Oct 1;52(10):1115-8.

19. Tsukise A, Sugawa Y, Okano M. Two anomalous cases of the right subclavian artery arising directly from the aortic arch in dogs. Nihon Juigaku Zasshi. 1972;34(1):11-5.

20. Donnelly LF, Fleck RJ, Pacharn P, Ziegler MA, Fricke BL, Cotton RT. Aberrant subclavian arteries. Am J Roentgenol [Internet]. 2002 May 1;178(5):1269-74. Available from: https://doi.org/10.2214/ajr.178.5.1781269

21. Cinà CS, Arena GO, Bruin G, Clase CM. Kommerell's diverticulum and aneurysmal rightsided aortic arch: A case report and review of the literature. J Vasc Surg. 2000;32(6):120814.

22. Saunders AB, Winter RL, Griffin JF, Mankin KMT, Miller MW. Surgical management of an aberrant left subclavian artery originating from a left patent ductus arteriosus in a dog with a right aortic arch and abnormal branching. J Vet Cardiol. 2013;15(2):153-9.

23. Smith J, Koury H, Hafner C, Welling R. Subclavian steal syndrome. A review of 59 consecutive cases. J. Thorac. Cardiovasc. Surg. 1994 Feb 1;35(1):11-4. Available from: http://europepmc.org/abstract/MED/8120071

24. Yamane $T$, Awazu T, Fujii Y, Watanabe T, Muto M, Okutsu M, et al. Aberrant branch of the bronchoesophageal artery resembling patent ductus arteriosus in a dog. J Vet Med Sci. 2001;63(7):819-22.

25. Fujii Y, Aoki T, Takano H, Ishikawa R, Wakao Y. Arteriovenous shunts resembling patent ductus arteriosus in dogs: 3 cases. J Vet Cardiol. 2009;11(2):147-51. 
26. Culshaw GJ, Wagner T, Fuentes VL, Schwarz T, Yool DA, French AT, et al. Identification and surgical ligation of aortopulmonic vascular malformation causing left heart volume overload in 4 dogs. J Vet Intern Med. 2013;27(3):583-7.

27. Ledda G, Caldin M, Mezzalira G, Bertolini G. Multidetector-row computed tomography patterns of bronchoesophageal hypertrophy and systemic-to-pulmonary fistula in dogs. Vet Radiol Ultrasoun. 2015;56(4):347-58.

28. Scollan K, Salinardi B, Bulmer BJ, Sisson DD. Anomalous left-to-right shunting communication between the ascending aorta and right pulmonary artery in a dog. J Vet Cardiol. 2011;13(2):147-52.

29. Markovic LE, Kellihan HB, Roldán-Alzate A, Drees R, Bjorling DE, Francois CJ. Advanced multimodality imaging of an anomalous vessel between the ascending aorta and main pulmonary artery in a dog. J Vet Cardiol. 2014;16(1):59-65.

30. Roux AL, Granger LA, Reynolds C, Gaschen L. Computed tomography features of bronchial and non-bronchial collateral arterial circulation development in a dog diagnosed with multiple chronic pulmonary thrombi. J Vet Cardiol. 2013;15(4):283-7.

31. Fong LV, Anderson RH, Siewers RD, Trento A, Park SC. Anomalous origin of one pulmonary artery from the ascending aorta: a review of echocardiographic, catheter, and morphological features. Brit Heart J. 1989;62(5):389.

32. Khan S, Nihill MR. Clinical presentation of persistent 5th aortic arch: 3 new cases. Texas Heart Institute journal [Internet]. 2006 Jan 1;33(3):361-4. Available from:

https://pubmed.ncbi.nlm.nih.gov/17041697

33. Gerlis LM, Dickinson DF, Wilson N, Gibbs JL. Persistent fifth aortic arch. A report of two new cases and a review of the literature. Int J Cardiol. 1987;16(2):185-92. 
Figures
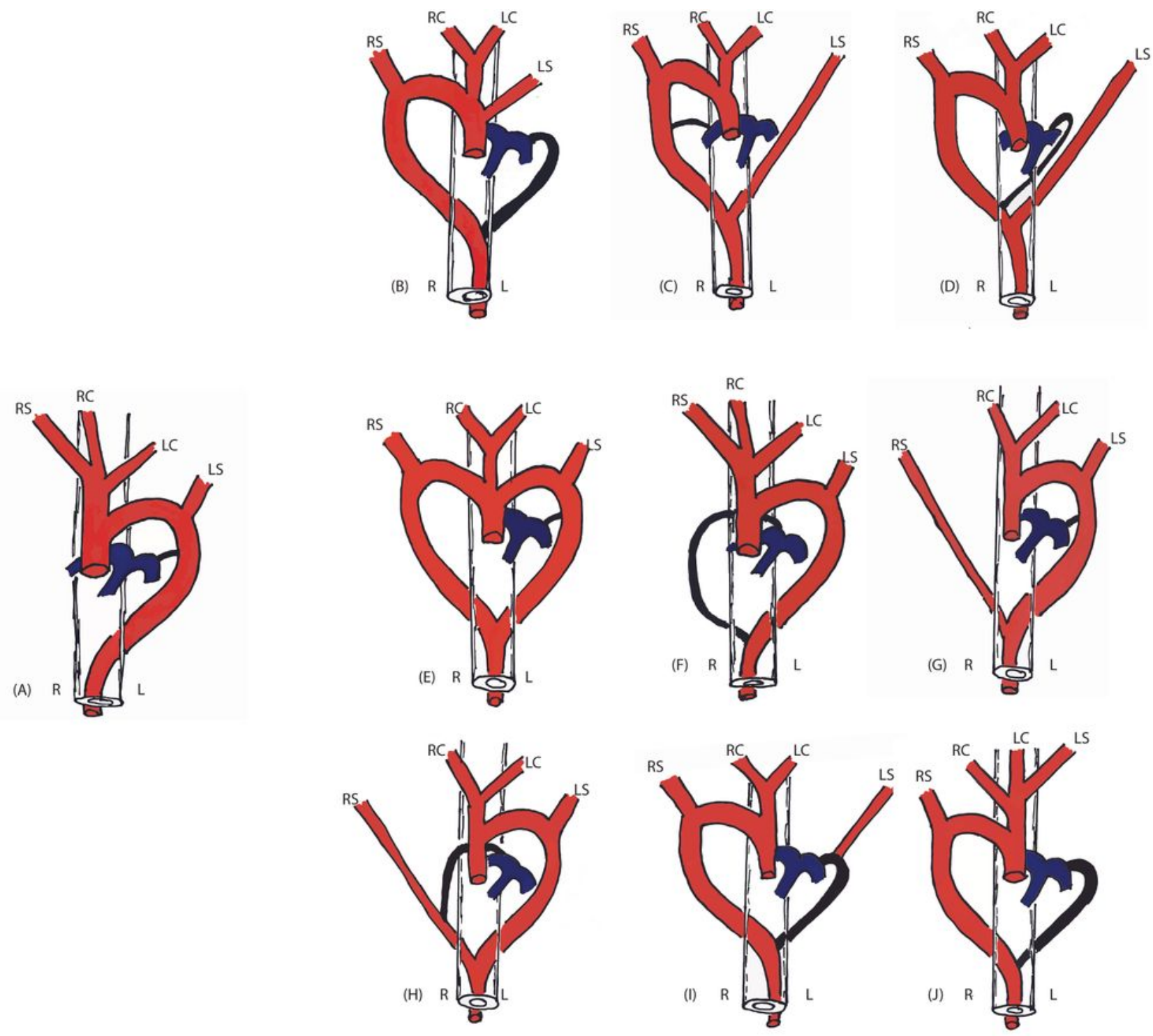

\section{Figure 1}

Classification scheme for aortic arch anomalies (A) normal anatomy of the aortic arch; (B) Type 1, consisting of persistent right aortic arch with persistent left ligamentum arteriosum; (C) Type 2, consisting of persistent right aortic arch with persistent left subclavian artery; (D) Type 3, consisting of persistent 
right aortic arch with persistent left ligamentum arteriosum and left subclavian artery; (E) Type 4, consisting of double aortic arch; (F) Type 5, consisting of normal left aortic arch with persistent right ligamentum arteriosum; (G) Type 6, consisting of normal left aortic arch with persistent right subclavian artery; $(\mathrm{H})$ Type 7, consisting of normal left aortic arch with persistent right ligamentum arteriosum and right subclavian artery; (I) Type 8, consisting of right aortic arch with left subclavian branching from the left sided ligamentum arteriosum; $(J)$ Type 9, consisting of right sided aortic arch with "mirror" image. 